\title{
Age-dependent biomechanical properties of the skin
}

\author{
Mariola Pawlaczyk ${ }^{1}$, Monika Lelonkiewicz ${ }^{1}$ Michat Wieczorowski \\ 1Department of Geriatrics and Gerontology, Poznan University of Medical Sciences, Poznan, Poland \\ Head of Department: Mariola Pawlaczyk MD, PhD \\ 2Institute of Mechanical Technology and Measurement Systems, Poznan University of Technology, Poznan, Poland \\ Head of Department: Michał Wieczorowski PhD
}

Postep Der Alergol 2013; XXX, 5: 302-306

DOI: $10.5114 /$ pdia.2013.38359

\begin{abstract}
The skin fulfills one of its most important functions, that is protection from mechanical injuries, due to the mechanism of reversible deformation of the structure. Human skin is a complex living material but in biomechanical tests it reveals its homogeneous nature. Biomechanical skin parameters change with time. Results of thickness measurements, where the skin was subjected to pressure, revealed that the Young's modulus increased linearly with age. The process of ageing is the reason why the skin becomes thinner, stiffer, less tense and less flexible. Skin tension measured during in vivo uniaxial load and the elasticity modulus are higher in children than in elderly adults. Furthermore, mean ultimate skin deformation before bursting is $75 \%$ for newborns and $60 \%$ for the elderly. Several types of the main lines were distinguished on the skin. The static lines, described by Langer, correspond to the lines of maximum tension, the Kraissl's lines correspond to the movements of the skin during muscle work, whereas the Borges lines are the relaxed skin tension lines. Biomechanical tests of the human skin help to quantify the effectiveness of dermatological products, detect skin diseases, schedule and plan surgical and dermatological interventions and treatments.
\end{abstract}

Key words: skin, biomechanics, ageing.

\section{Introduction}

The human skin protects the organism from the external environment, most importantly from mechanical injuries, what is enabled by the mechanism of reversible deformation of the skin structure [1]. All tissues, including skin, undergo deformation under the influence of external forces, particularly the weight. Human skin can be stretched to several times its original size and still maintains its original phenotypic properties [2-4]. Such impressive expansion is possible because the skin is a highly specialized mechanical structure, responding through a network of interconnected cascades of chemical reactions, with the participation of extracellular, cytoplasmic and nuclear membranes [5]. When the skin is stretched above its physiological limit, a series of reactions activating ion channels, integrins, growth factor receptors and G-receptors conjugated with protein reactions takes place. These reactions aim to restore the homeostatic balance [6]. The same response occurs in mechanical skin damages related to esthetic dermatology and plastic surgery [7]. Integrins mediate between the cell and the cellular matrix, playing the key role in the strength of transmission through the cell membranes of e.g. signaling modules related to mitogen-activated protein kinase (MAPK), nitric oxide (NO) and phosphoinositide-3-kinase (PI3K). Therefore, mechanical activation of the skin initiates the signaling pathways, which in turn activate the transcription of factors stimulating gene expression. That causes a cascade of events which results in an increased mitotic activity and collagen synthesis [6-8]. The skin also has impressive functional plasticity which allows for its progressive adaptation to the environment. Changes in the skin tissue occurring during dermatological and surgical treatments initiate mechanotransductive paths that also increase the mitotic activity and the synthesis of collagen [9]. However, if external stimuli such as mechanical stress reach sufficiently large values, they may cause irreversible deformation and damage to the skin, resulting in a loss of its mechanical properties $[10,11]$.

Human skin is a complex living material, composed of several heterogeneous layers: epidermis, dermis and subcutaneous tissue, which is sticky and soft $[1,12,13]$. Skin

Address for correspondence: Mariola Pawlaczyk MD, PhD, Department of Geriatrics and Gerontology, Poznan University of Medical Sciences, 5 Święcickiego St, 60-781 Poznan, Poland, phone: +48 6185465 85, e-mail: mariolapawlaczyk@o2.pl Received: 10.02.2013, accepted: 22.05.2013. 
thickness varies depending on its anatomic location, fluid content and age [14]. From the mechanical point of view, the skin is a very complex structure. Despite the fact that it consists of three layers with different mechanical properties, in medical tests the skin reacts as a homogeneous material and is treated as such in all indentation measurements [15]. Analyses of skin morphology using quantitative methods of mechanical engineering are carried out in studies on skin ageing. This allows to determine the level of mechanical skin changes progressing with age. Physiological functions of the skin can be determined by mechanical parameters, which are subject to change, depending on the density and elastic components of the dermis [16].

\section{Skin elasticity}

Deformation marks the skin in response to applied forces and is defined as perfectly elastic, if the skin returns to its initial state after the termination of the force. If, after exceeding the elastic limit, the termination of the external force does not permit for the skin to return to its initial shape, the so-called residual deformation occurs. This deformation is related to the change of position of the skin elements and, consequently, its stability. Elastic potential energy appears as a result of the interaction between the skin elements [17]. The skin, being an elastic material, is subject to the mechanical laws defining its properties. The modulus of longitudinal elasticity - the Young's modulus $(E)$ - defines the relation between stress $(\sigma)$ and strain $(\varepsilon)$ in the skin. The modulus characterizes skin resistance to elastic elongation. This dependence is generally presented by the Hooke's Law: $\varepsilon=E / \sigma[15]$. In the elastic range the relationship between stress and strain is linear and the factor of proportionality is expressed by the Young's modulus. The unit of Young's modulus is the Pascal ( $\left.\mathrm{Pa}, \mathrm{N} / \mathrm{m}^{2}\right)$. The Young's modulus is defined as: $E=\operatorname{tg} \alpha$ [17-20]. In the literature, the Young's modulus $(E)$ of the skin fluctuates between 0.42 MPa and 0.85 MPa [1, 20] for the torsion tests, between 4.6 MPa and 20 MPa [21] when extracting stress values obtained in tests carried by means of mechanical equipment, and between $0.05 \mathrm{MPa}$ and $0.15 \mathrm{MPa}$ [1, 20, $22,23]$ in the suction tests. Large discrepancies in the results may show evidence for the changes occurring in the skin during the process of ageing, as well as the differences in the skin properties, depending on its anatomic location.

Results of skin thickness measurements, where the skin was subjected to pressure, obtained in ultrasound tests in comparison to a theoretical module revealed that the Young's modulus increased linearly with age. In the process of ageing, the skin becomes thinner, stiffer, less tense and less flexible [24]. Ultrasound images showed that thickness of the epidermis and dermis of elderly people is about 0.7$0.8 \mathrm{~mm}$ smaller in comparison to normal skin [25, 26]. Figure 1 illustrates the characteristics of skin resistance [27]. Skin resistance is described by yield limit and elongation of the material. Plastic elongation ( $A r)$ is calculated using the following formula: $\mathrm{Ar}=(\mathrm{L} 1-\mathrm{LO}) / \mathrm{LO} \times 100 \%$, where $\mathrm{LO}$ stands for the section of the sample before deformation and L1 stands for the section of the sample after deformation. Yield point is a point of stress when notable plastic deformations become visible [2, 10, 11, 19, 28, 29]. Skin biomechanics defines also the Poisson's ratio (coefficient) which determines proportionality of mutually perpendicular linear elongations. It is shown by the following equation: $\Delta a / a=\mu \Delta / /$, where $\alpha$ and $l$ are lengths of the sides which are subject to linear expansion activities. The equation shows that stretching is followed by elongation of the skin in the direction of the acting forces and reduction in the transverse dimensions, whereas compression is followed by reduction in the longitudinal dimensions and extension of the transverse dimensions [18, 30].

Skin parameters change with age. The study on skin stretching by Vogel et al. identified age-related differences. Skin tension which was measured during the in vivo uniaxial load in a child was about $21 \mathrm{~N} / \mathrm{mm}^{2}(\mathrm{MPa})$, while the result measured in an elderly adult decreased to about $17 \mathrm{~N} / \mathrm{mm}^{2}$ (MPa). The child elasticity modulus had an average of $70 \mathrm{~N} / \mathrm{mm}^{2}(\mathrm{MPa})$, while the elderly adult elasticity modulus had an average of $60 \mathrm{~N} / \mathrm{mm}^{2}$ (MPa). Furthermore, mean ultimate skin deformation before bursting was $75 \%$ for newborns and $60 \%$ for the elderly [31, 32].

Obviously, the mathematical description requires data about mechanical properties of the skin. They are obtained during strength tests that determine deformation of the skin under various external conditions, when the skin strength is inspected using increasing load to get a function. The results describe average mechanical properties of the skin. Basic law of strength says that material undergoes deformation if stress is applied. There are two types of deformations: elastic deformation, which disappears after the removal of the load, and permanent deformation, also called plastic, which remains after the removal of the stress. Permanent deformations accompany the process of applying

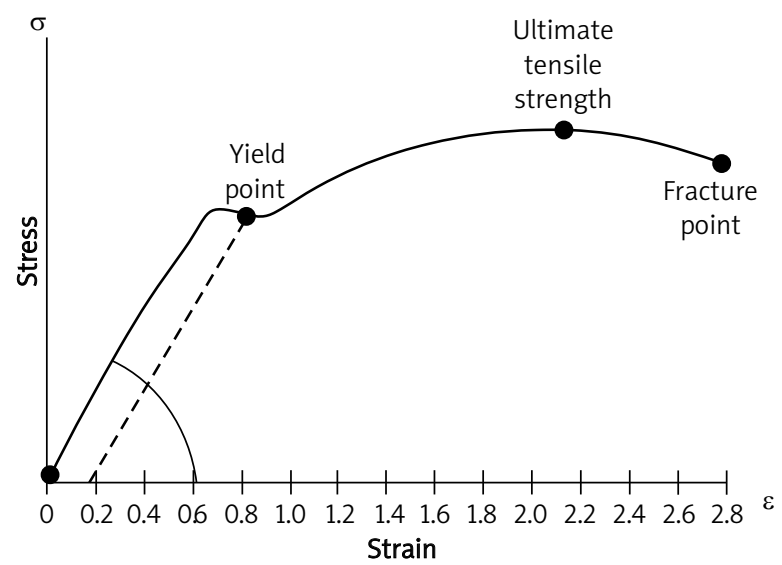

Figure 1. Stretching of elastic-plastic material [28] 
A

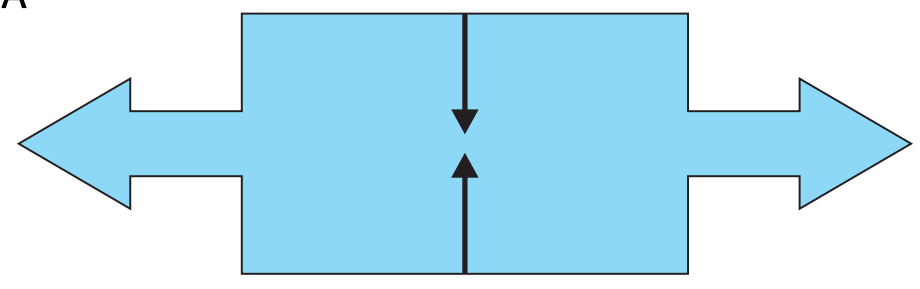

B

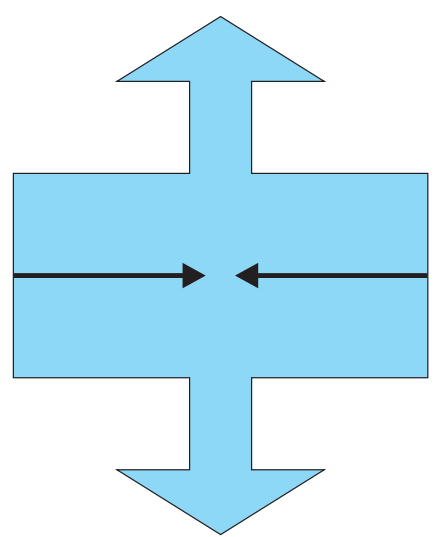

Figure 2. Linear elongation in the skin depending on the vector of the working force. A-Distribution of the forces during skin stretching when its thickness is constant. B - Distribution of the forces during skin squeezing when its thickness is constant

stress from the very beginning, however, in the case of linear elastic material such as skin, their power begins to have practical significance after exceeding the size known as the elastic limit as shown in Figure 1 [28].

\section{Linear elasticity}

During stretching, the distance between skin components increases and its molecule energy rises. Similarly, during compression of the skin, the distance between its components decreases and its energy rises, causing imbalance. Forces causing the reduction of distance between skin components, in other words attractive forces, appear in the process of stretching, whereas forces causing an increase in the distance between skin components, or repulsive forces, appear in the process of compression [17, 30]. These forces balance the external forces influencing the skin; $p=F / S$, where: $p$ - stress vector, $F$ - force vector, $S$ - surface perpendicular to $F$. If, as a result of external forces, there is a change in one dimension of the skin, we deal with onesided deformation. While stretching, the skin changes its length $\Delta \lambda>0$ and when compressing $\Delta \lambda<0$. Appropriate relative elongation while stretching is $\Delta \lambda / \lambda>0$, and while squeezing $\Delta \lambda / \lambda<0$. Unilateral deformation is an idealized case because spatial displacement of the skin components, related to the change of its volume, occurs as a result of the applied forces. Thus, the change in volume $\Delta \lambda$ characterizes the actual deformation $[17,30]$. During stretching the skin is prolonged in the direction of the acting forces and the transverse dimension or thickness are reduced, whereas during compression the longitudinal dimensions are shorted and the transverse dimensions are prolonged or skin thickness increases (Figures $2 \mathrm{~A}$ and B) $[16-18,30]$. The vector of skin tension in the linear treatments with hyaluronic acid injections, for example hyaluronic threads or fern pattern technique, depends on skin thickness and forces associated with the injection of the hyaluronic acid [33].

\section{Anisotropy}

The skin and subcutaneous tissue provide a protective covering of the body, capable of stretching and contracting. Theoretically, these processes may occur in any direction and are mutually inclusive. In practice, the skin shows anisotropic properties and its ability to stretch and contract in different directions varies. Anisotropy increases with age [34]. Skin tensions depend on the mutual position of the fibers subjected to the external forces, whereas mechanical properties of the skin are dependent on the force vector applied [17, 35]. Microscopic observations of human skin revealed that skin topography has a network of lines. The organization of the lines reflects multidirectional tensions connected with its construction. This morphology is present at birth and its scale of depth and wavelength increases with age [36]. Two types of lines on the skin area have been described. The main lines, formed in the superficial layer of the dermis, correspond to the distribution of tension forces in the skin - they are visible in the dermis even after the epidermis is separated. They depend on the distribution of tensions in the collagen and elastic fibers, as well as the extracellular matrix - including the hyaluronic acid. The secondary lines are of epidermal origin [37]. The system of the main lines is related with the distribution of collagen and elastic fibers. Both types of fibers form a structural and functional integrity. The role of elastin is to restore the location of collagen. Reticular layer of the dermis contains elastin arranged in thick, horizontal fibers. The papillary layer of the dermis, which is thinner, contains elastic fibers arranged perpendicular to the skin surface, what enables their binding by intercalation of microfibrils with the dermal-epidermal border. The extensive network of elastin provides elasticity for all layers of the skin, while collagen is the protein responsible for skin firmness [38]. There are at least sixteen types of collagen, with types number I, II and III representing about 
$80-90 \%$ of the collagen in the body $[39,40]$. Tropocollagen (300 nm long and $1.5 \mathrm{~nm}$ in diameter) is the basic unit of the collagen fiber. This protein has three spiral subunits that coil around each other to form a fiber. The diameter of collagen fibers ranges from $50 \mathrm{~nm}$ to $200 \mathrm{~nm}$. They in turn form fiber bundles, whose diameter is $0.5-3 \mathrm{~mm}$ [40]. In the healthy skin the fiber bundles run parallel to the epidermis surface and are connected by thin strands of collagen fibers. Studies using the method of spectral analysis (the fast Fourier test, FFT) revealed a much higher anisotropy of the healthy skin in comparison to the keloids, which demonstrated features of isotropy. This result confirms the existence of an organized and specifically targeted system of fibrous bundles in healthy tissues [40, 41]. Several types of the main lines were distinguished. The static lines were described by Langer and correspond to the lines of maximum tension [42]. The dynamic lines were described by Kraissl and Borges [43]. The Kraissl's lines correspond to the movements of the skin during muscle work and the Borges lines, which are presented in Figure 3, are the relaxed skin tension lines (RSTL) [44]. Collagen fibers are arranged with their long axis parallel to the reduced skin tension lines, which are visible on the surface of the skin as the main lines $[40,45,46]$. Skin tension lines are accompanied by constant points and lines. In fact, the reduced skin tension lines have their origin in them. It was noted that they begin in constant points and run parallel to the constant lines [47]. They can be determined during movement, for example during facial movement. It is important to determine and mark the main lines and constant points when planning a surgical operation, dermatosurgical and esthetic dermatology treatment. In surgery and dermatosurgery, an appropriate direction of a simple skin incision, and most of all complex cuts connected with the production of local skin flaps, will determine the final functional and cosmetic outcome. In 1970, Thacker et al. emphasized the importance of skin biomechanics in plastic surgery, concluding that the direction of cuts should be correlated with the direction of reduced tension in the skin, resulting in more narrow and less visible scars $[48,49]$. All properties of the skin described in this article characterize the skin under physiological conditions. In the case of esthetic dermatology treatments, after intradermal injections of fillers such as the hyaluronic acid, we deal with composite material, in other words, material made up of at least two different components, whose connection occurs at the macroscopic level. This is elastic-plastic material with reinforcement. The components of the composite material can be defined as the continuous phase (the matrix) - in this case the skin, and the dispersed phase surrounded by the matrix - the hyaluronic acid.

\section{Conclusions}

Biomechanical parameters of the skin alter in the course of human life. During the process of ageing the skin be-

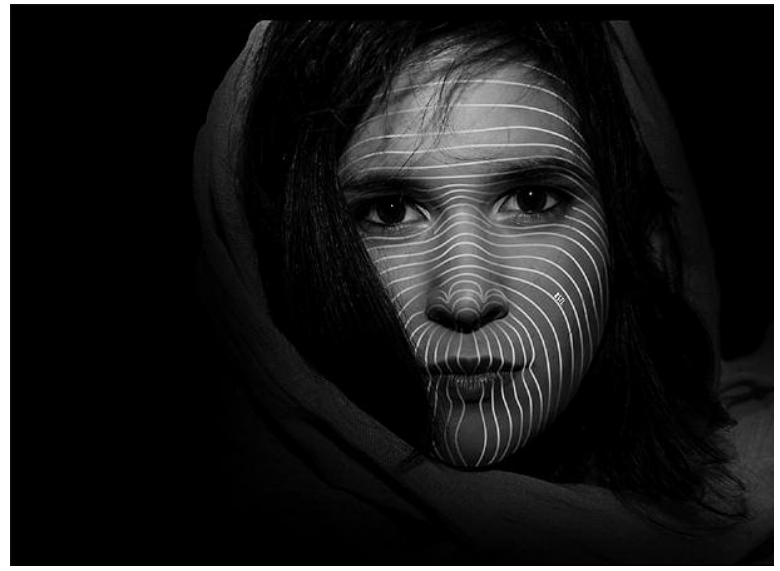

Figure 3. Relaxed skin tension lines also described as Borges lines

comes thinner, stiffer, less tense and less flexible, what lowers its protective functions against mechanical injuries. Biomechanical studies on human skin contribute substantially to quantifying the efficiency of various dermatological products, detecting skin diseases, as well as planning surgical and dermatological interventions.

\section{References}

1. Pailler-Mattei C, Beca S, Zahouani H. In vivo measurements of the elastic mechanical properties of human skin by indentation tests. Med Engin Phys 2008; 30: 599-606.

2. De Filippo RE, Atala A. Stretch and growth: the molecular and physiologic influences of tissue expansion. Plast Reconstr Surg 2002; 109: 2450-62.

3. Sanders JE, Goldetein BS, Leotta DF. Skin response to mechanical stress: adaptation rather than breakdown - a review of the literature. J Rehab Res Develop 1995; 32: 214-26.

4. Silver FH, Seehra GP, Freeman JW, Devore D. Viscoelastic properties of young and old human dermis: a proposed molecular mechanism for elastic energy storage in collagen and elastin. J Appl Polymer Sci 2002; 86: 1978-85.

5. Silver FH, Siperko LM, Seehra GP. Mechanobiology of force transduction in dermal tissue. Skin Res Technol 2003; 9: 3-23.

6. Wong VW, Akaishi S, Longaker MT, Gurtner GC. Pushing back: wound mechanotransduction in repair and regeneration. J Invest Dermatol 2011; 131: 2186-96.

7. Simpson CL, Patel DM, Green KJ. Deconstructing the skin. Cytoarchitectural determinants of epidermal morphogenesis. Nat Rev Mol Cell Biol 2011; 12: 565-80.

8. Takei T, Mills I, Arai K, Sumpio BE. Molecular basis for tissue expansion: clinical implications for the surgeon. Plast Reconstr Surg 1998; 102: 247-58

9. Zollner AM, Buganza Tepole A, Kuhl E. On the biomechanics and mechanobiology of growing skin. J Theor Biol 2012; 297: 166-75.

10. Buganza Tepole A, Gosain AK, Kuhl E. Stretching skin - the physiological limit and beyond. Int J Nonlin Mech 2012; 47: 938-49.

11. Buganza Tepole A, Ploch CJ, Wong, J, et al. Growing skin: a computational model for skin expansion in reconstructive surgery. J Mech Phys Solids 2011; 59: 2177-90. 
12. Wu JZ, Dong RG, Smutz WP, Schopper AW. Modeling of time dependent force response of fingertip to dynamic loading. J Biomech 2003; 36: 383-92.

13. Kathyr F, Imberdis C, Vescovo P, et al. Model of the viscoelastic behaviour of skin in vivo and study of anisotropy. Skin Res Technol 2004; 10: 93-103.

14. Agache P. Physiologie de la peau et exploitations fonctionnelles cutanees. Tec et Doc Lavasier, Paris 2001.

15. Boyer G, Laquieze L, Le Bot A, et al. Dynamic indentation on human skin in vivo: ageing effects. Skin Res Technol 2009; 15: 55-67.

16. Zahouani H, Pailler-Mattei C, Sohm B, et al. Characterization of the mechanical properties of a dermal equivalent compared with human skin in vivo by indentation and static friction tests. Skin Res Technol 2009; 15: 68-76.

17. Meijer R, Douven LFA, Oomens CWJ. Characterisation of anisotropic and non-linear behaviour of human skin. Comput Methods Biomech Biomed Engin 1999; 2: 13-27.

18. Tilleman TR, Tilleman MM, Neumann MHA. The elastic properties of cancerous skin: poisson's ratio and Young's modulus. Isr Med Assoc J 2004; 6: 753-5.

19. Lee EH. Elastic-plastic deformation at finite strains. J Appl Mech 1969; 36: 1-6.

20. Agache PG, Monneur C, Leveque JL, de Rigal J. Mechanical properties and Young's modulus of human skin in vivo. Arch Dermatol Res 1980; 269: 127-33.

21. Manschot JF, Brakkee AJ. The measurement and modelling of the mechanical properties of human skin in vivo. I. The measurement. J Biomech 1986; 19: 511-5.

22. Diridollou S, Patat F, Gens F, et al. In vivo model of the mechanical properties of the human skin under suction. Skin Res Technol 2000; 6: 214-21.

23. Hendriks FM, Brokken D, Van Eemeren J, et al. A numericalexperimental method to characterize the non linear mechanical behaviour of human skin. Skin Res Technol 2003; 9: 27483.

24. Diridollou S, Vabre V, Berson M, et al. Skin ageing: changes of physical properties of human skin in vivo. Int J Cosmet Sci 2001: 23: 353-62.

25. Kaya G, Saurat JH. Dermatoporosis: a chronic cutaneous insufficiency/fragility syndrome. Dermatology 2007; 215: 284-94.

26. Saurat JH. Dermatoporosis, the functional side of skin agening. Dermatology 2007; 215: 271-2.

27. Roylance D. Stress- strain diagram. http//www.websters-dictionary-online.com

28. Holt B, Tripathi A, Morgan J. Viscoelastic response of human skin to low magnitude physiologically relevant shear. J Biomech 2008; 41: 2689-95.

29. Zeng Y, Liu Y, Xu Ch, et al. Biomechanical properties of skin in vitro for different expansion methods. Clin Biomech (Bristol, Avon) 2004; 19: 853-7.

30. Demtroeder W. Experimentalphysik 1. Springer, Berlin-Heidelberg 2006

31. Vogel HG. Age dependence of mechanical and biochemical properties of human skin. Part I. Stress-strain experiments, skin thickness and biochemical analysis. Bioeng Skin 1987; 3: 67-91.

32. Vogel HG. Age dependence of mechanical and biochemical properties of human skin. Part II. Hysteresis, relaxation, creep and repeated strain experiments. Bioeng Skin 1987; 3: 41-176.

33. van Eijk T, Braun M. A novel method to inject hyaluronic acid: the Fern Pattern Technique. J Drugs Dermatol 2007; 6: 805-8.

34. Żak M, Szotek S, Kuropka P, et al. Determination of the mechanical properties of the skin of foetuses with respect to its structure. Acta Bioeng Biomech 2011; 13: 37-4.
35. Ní Annaidha A, Bruyèred K, Destradea M, et al. Characterization of the anisotropic mechanical properties of excised human skin J Mech Behav Biomed Mater 2012; 5: 139-48.

36. Zahouani H, Boyer G, Pailler-Mattei C. Effect of human ageing on skin rheology and tribology. Wear 2011; 271: 2364-9.

37. Lagarde JM, Rouvrais C, Black D. Topography and anisotropy of the skin surface with ageing. Skin Res Technol 2005; 11: 110-9.

38. Kielty CM, Sherratt MJ, Shuttleworth CA. Elastic fibres. J Cell Sci 2002; 115: 2817-28.

39. Lodish H, Berk A, Zipursky SL, et al. Molecular cell biology. $4^{\text {th }}$ edn. WH Freeman, New York 2000; 979-85.

40. Cicchi R, Kapsokalyvas D, De Giorgi V, et al. Scoring of collagen organization in healthy and diseased human dermis by multiphoton microscopy. J Biophoton 2010; 3: 34-43.

41. English RS, Shenefelt PD. Keloids and hypertrophic scars. Dermatol Surg 1999; 25: 631-8.

42. Bush J, Ferguson MWJ, Mason T, McGrouther G. The dynamic rotation of Langer's lines on facial expression. J Plast Reconstr Aesthet Surg 2007; 60: 393-9.

43. Kraissl CJ, Conway H. Excision of small tumours of the skin of the face with special reference to the wrinkle lines. Surgery 1949; 4: 592-600.

44. Borges AF. Relaxed skin tension lines (RSTL) versus other skin lines. Plast Reconstr Surg 1984; 73: 144-50.

45. Waldorf JC, Perdikis G, Terkonda SP. Planning incisions. Oper Tech Gen Surg 2002; 14: 199-206.

46. Sakai S, Yamanari M, Lim Y, et al. In vivo evaluation of human skin anisotropy by polarization-sensitive optical coherence tomography. Biomed Optic Express 2011; 2: 24-6.

47. Illouz YG. Surgical implications of "fixed points": a new concept in plastic surgery. Aesth Plast Surg 1989; 13: 137-44.

48. Thacker JG, Stalnecker MC, Allaire PE, et al. Practical applications of skin biomechanics. Clin Plast Surg 1977; 4: 167-71.

49. Seo H, Kim S, Cordier F, et al. Estimating dynamic skin tension lines in vivo using 3D scans. Computer-Aided Design 2013; 45: 551-5. 